\title{
Dynamic effective potential for $\alpha$-particle bound and quasibound states
}

\author{
C. H. Dasso, ${ }^{1}$ R. J. Liotta, ${ }^{2}$ and M. Lozano ${ }^{3}$ \\ ${ }^{1}$ The Niels Bohr Institute, University of Copenhagen, Blegdamsvej 17, DK-2100 Copenhagen , Denmark \\ ${ }^{2}$ Royal Institute of Technology, Frescativägen 24, S-10405 Stockholm, Sweden \\ and Nordita, Blegdamsvej 17, DK-2100 Copenhagen Ф, Denmark \\ ${ }^{3}$ Departamento de Física Atómica, Molecular y Nuclear Universidad de Sevilla, AP. 1065, E-41080 Sevilla, Spain
}

(Received 21 November 1995)

\begin{abstract}
We exploit analytic properties of the optical potential for elastic scattering of $\alpha$ particles on nuclei to extract information on the effective interaction that should be used to describe the motion of a cluster of two neutrons and two protons bound to a nuclear system. This prescription solves long-standing ambiguities in the formalisms used for the study of $\alpha$ decay. [S0556-2813(96)01308-8]

PACS number(s): 24.10.Ht, 21.60.Gx, 23.60.+e, 27.80.+w
\end{abstract}

Alpha decay was one of the first nuclear phenomena observed in nature. Not surprisingly, attempts to describe this process go back to the earliest days of quantum mechanics. Perhaps the most transparent formulation was that of Gamow [1], who recognized the possibilities of quantal tunneling to explain the many orders of magnitude spanned by the experimentally measured lifetimes. As the general knowledge of nuclear structure improved, however, this simple picture ran into troubles. These primarily had to do with the need to understand microscopically the formation inside of the mother nucleus of an alpha particle as a cluster of two protons and two neutrons. This goal introduced all kinds of difficulties in the problem, ranging from complex coupling schemes down to the treatment of the continuum required to satisfy the appropriate boundary conditions. Especially noteworthy are questions raised about the viability of a scheme that requires the wave function of the alpha particle not only at the nuclear surface, but in the interior as well [2]. The reservations center here mostly on the inhibiting role of the Pauli principle, which, inevitably, entered into the picture as soon as the alpha particle was thought of in terms of its constituent nucleons.

In this paper we return to this question following a path that aims to bypass these difficulties. To this end we rely on the work by Bell and Squires [3] (cf. also Ref. [4]) who investigated the analytic properties of the complex nucleonnucleus interactions. In their contribution they clarified the intimate connection between the optical potential for scattering states and the effective interaction that binds the nucleons to the core. In fact, they established the conceptual identity of these quantities, to the extent that the latter is none other than the analytic continuation of the former into the negative (or, more precisely, bound-state) energy domain.

When Bell and Squires presented their results, much was already known about the binding potential for single nucleons. This information had been mainly extracted from the analysis of nuclear systems either directly available in nature or artificially produced. Thus it could be argued that their work was mostly of academic interest. Their conclusions are, however, potentially much more relevant in the case of alpha particles. Michel et al. [5] and Delbar et al. [6] made significant steps in the understanding of the optical potential for the scattering of alpha particles by lighter systems, such as oxygen and calcium. Microscopic estimation of the static potential between an $\alpha$ particle and a nuclear core can also be contemplated by folding procedures; see, for instance, Refs. $[7,8]$. Uncertainties remain, however, about the couplings that should be used to account for the motion of the fournucleon cluster in a quasibound state of a heavier nucleus that eventually $\alpha$ decays. In fact, to calculate microscopically the renormalization of the bare interaction that gives rise to the actual energy dependence of the $\alpha$-nucleus potential under these circumstances is a rather formidable task. One can inspect, for instance, in Ref. [9], the numerous diagrams that have to be taken into account to construct in a perturbative approach the correlation and polarization terms that dynamically "dress" the mass operator for ordinary nucleons. Needless to say, the situation would get even more complicated in the case of alpha particles.

The prospects are vastly improved, however, if one takes a phenomenological approach in which the energy dependence of the optical potential is used. Indeed, an extrapolation of the empirically determined couplings to negative energies, if feasible, would yield a binding potential where Pauli blocking, surface effects, and other relevant corrections are properly included to all perturbative orders.

It is not necessary to "propose" such measurements since experimental studies of asymptotically free states have been available for a long time. In fact, one can simply go back to the literature and search for existing optical-model fits to the elastic cross sections of $\alpha$ particles on nuclei. We shall henceforth focus our attention only on the real part $V$ of the interaction, as it is expected that this component will have a smoother transition from positive to negative energies. ${ }^{1} \mathrm{We}$ show in Table I a collection of optical model parameters for

\footnotetext{
${ }^{1}$ Effective interactions get contributions related to nonlocality in space and time. It is well known that the energy dependence of the real part of the latter, $\Delta V$, has a counterpart in the imaginary part $W$. The connection between these two functional dependences for heavy ions has been successfully approached in terms of dispersion relations (see, for instance, Ref. [10]). The characteristic shape of $W(E)$ for $E \approx 0$, however, is not suitable for extrapolation.
} 
TABLE I. Real potential depth $V_{0}$, radius parameter $r_{0}\left(A_{T}^{1 / 3}\right)$, and diffuseness $a$ for a Woods-Saxon geometry as a function of bombarding energy $E$ in the elastic scattering of $\alpha$ particles by ${ }^{208} \mathrm{~Pb}$. Each row gives results for optical-potential fits from different experiments, as quoted in the corresponding references.

\begin{tabular}{|c|c|c|c|c|}
\hline$E(\mathrm{MeV})$ & $V_{0}(\mathrm{MeV})$ & $r_{0}(\mathrm{fm})$ & $a(\mathrm{fm})$ & Ref. \\
\hline 16.0 & 35.0 & 1.550 & 0.570 & [22] \\
\hline 18.0 & 35.0 & 1.550 & 0.570 & [23] \\
\hline 19.0 & 96.4 & 1.376 & 0.625 & [24] \\
\hline 19.5 & 35.0 & 1.550 & 0.570 & [27] \\
\hline 20.0 & 96.4 & 1.376 & 0.625 & [24] \\
\hline 20.0 & 174.0 & 1.470 & 0.470 & [27] \\
\hline 20.0 & 35.0 & 1.550 & 0.570 & [23] \\
\hline 21.0 & 32.0 & 1.550 & 0.570 & [27] \\
\hline 22.0 & 96.4 & 1.376 & 0.625 & [24] \\
\hline 22.0 & 35.0 & 1.550 & 0.570 & [27] \\
\hline 22.0 & 94.6 & 1.364 & 0.650 & [24] \\
\hline 22.0 & 117.5 & 1.298 & 0.700 & [24] \\
\hline 22.0 & 86.9 & 1.449 & 0.550 & [24] \\
\hline 22.0 & 159.7 & 1.387 & 0.560 & [24] \\
\hline 22.0 & 110.9 & 1.464 & 0.500 & [24] \\
\hline 22.0 & 35.0 & 1.518 & 0.571 & [23] \\
\hline 22.0 & 92.5 & 1.384 & 0.625 & [24] \\
\hline 22.0 & 100.4 & 1.444 & 0.542 & [24] \\
\hline 22.0 & 90.3 & 1.411 & 0.600 & [24] \\
\hline 22.0 & 200.2 & 1.390 & 0.529 & [24] \\
\hline 22.5 & 33.0 & 1.550 & 0.570 & [27] \\
\hline 23.5 & 117.5 & 1.298 & 0.700 & [25] \\
\hline 23.5 & 86.9 & 1.449 & 0.550 & [25] \\
\hline 23.5 & 92.5 & 1.384 & 0.625 & [25] \\
\hline 23.5 & 110.9 & 1.464 & 0.500 & [25] \\
\hline 24.5 & 37.0 & 1.550 & 0.570 & [27] \\
\hline 25.5 & 35.0 & 1.550 & 0.570 & [23] \\
\hline 25.5 & 41.0 & 1.550 & 0.570 & [27] \\
\hline 27.0 & 98.4 & 1.371 & 0.621 & [22] \\
\hline 96.0 & 89.3 & 1.350 & 0.710 & [26] \\
\hline 104.0 & 60.0 & 1.392 & 0.656 & [27] \\
\hline 129.0 & 89.3 & 1.350 & 0.710 & [26] \\
\hline 139.0 & 110.0 & 1.315 & 0.705 & [27] \\
\hline 139.0 & 155.0 & 1.282 & 0.677 & [27] \\
\hline 139.0 & 200.0 & 1.261 & 0.657 & [27] \\
\hline 166.0 & 119.0 & 1.260 & 0.740 & [27] \\
\hline 172.0 & 155.0 & 1.282 & 0.677 & [26] \\
\hline 218.0 & 119.9 & 1.260 & 0.740 & [26] \\
\hline 340.0 & 67.2 & 1.325 & 0.800 & [28] \\
\hline 480.0 & 63.0 & 1.300 & 0.950 & [28] \\
\hline
\end{tabular}

the specific reaction $\alpha+{ }^{208} \mathrm{~Pb}$. It may be somewhat puzzling at first to see the diversity of depths, radii, and diffuseness values that such a compilation reveals. One must, however, recognize that the numbers represent completely independent efforts where no attempt was made to put in evidence an overall energy dependence of the interaction.

It is clear that our first task is then to reprocess the information contained in Table I, so as to establish a point of contact between the different measurements. There are several ways to incorporate the energy dependence of the inter-

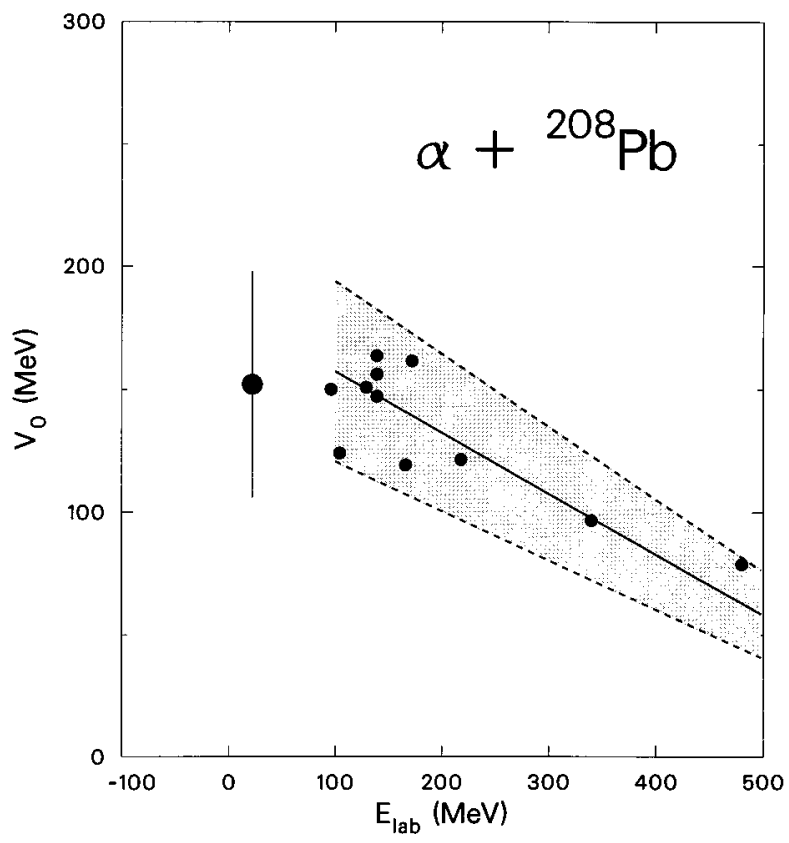

FIG. 1. Potential depth $\bar{V}_{0}(E)$ extracted from the experiments quoted in Table I and converted to a common Woods-Saxon geometry with radius parameter $r_{0}=0.98 \mathrm{fm}$ and diffuseness $a=0.80 \mathrm{fm}$. The solid line gives the energy dependence suggested in Ref. [1], while the dashed lines give the experimental margins defined by the error bars.

action. We choose, for our present purpose, to define a common Woods-Saxon geometry

$$
V(r)=-\frac{\bar{V}_{0}}{1+\exp [(r-R) / a]},
$$

where $R=r_{0}\left(208^{1 / 3}+4^{1 / 3}\right)$, and to exhibit the dynamical content of the effective interaction in terms of an energydependent strength $\bar{V}_{0}=\bar{V}_{0}(E)$. For the radius and diffuseness parameters, we take $r_{0}=0.98 \mathrm{fm}$, and $a=0.80 \mathrm{fm}$. This choice is inspired by the convenient parametrization of the energy dependence of the interaction given in [11], namely,

$$
V_{0}(E)=(182.1 \pm 41.3)+(-0.248 \pm 0.047) E,
$$

which is valid for $E>80 \mathrm{MeV}$. Comparing potentials at a strong-interaction radius (in fm) $r_{s}=1.07\left(208^{1 / 3}\right.$ $\left.+4^{1 / 3}\right)+2.72-a \ln \left[1+2\left(E_{\mathrm{c}, \mathrm{m} .}-E_{b}\right) / E_{b}\right]$, where $E_{b}$ is the Coulomb barrier [12], we have extracted from the opticalmodel parameters listed in Table I the potential depths $\bar{V}_{0}(E)$. These are plotted in Fig. 1; reassuringly, all points now fall in a rather ordered pattern. The straight lines correspond to the parametrization (2), allowing for the uncertainty in the coefficients. For the many data points at $E \approx 20 \mathrm{MeV}$, we have opted to show their span in $\bar{V}_{0}$, which fits quite well within the trend exhibited at other bombarding energies.

The choice of ${ }^{208} \mathrm{~Pb}$ as a target nucleus was geared to the eventual investigation of the radioactive $\alpha$ decay of ${ }^{212} \mathrm{Po}$. The separation energy of an alpha particle from this nucleus is about $8 \mathrm{MeV}$. It follows from the systematics displayed in Fig. 1 that the $\alpha^{-208} \mathrm{~Pb}$ potential in this energy range can be approximated by 


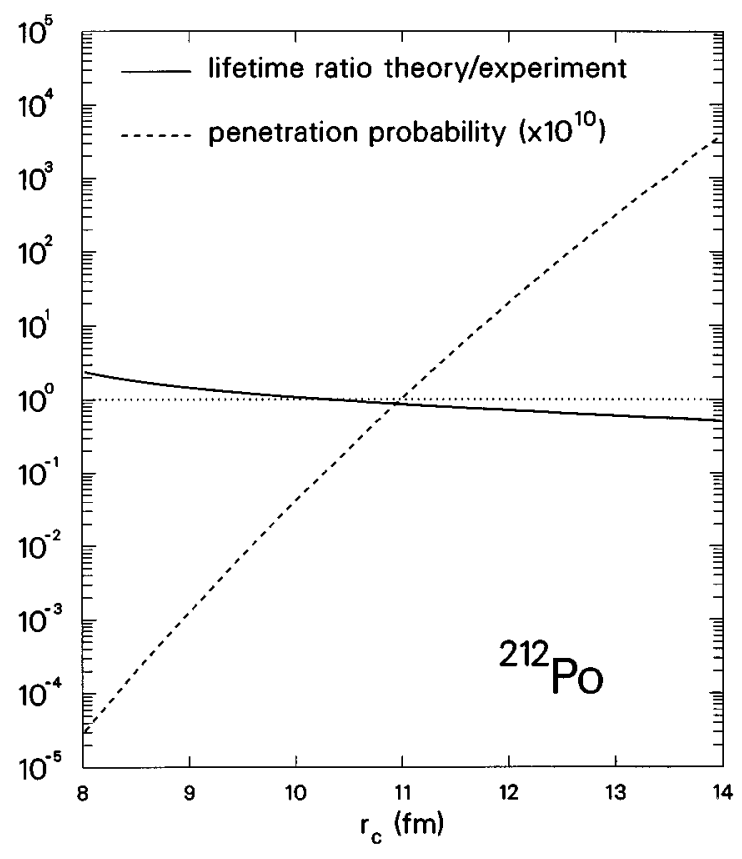

FIG. 2. Lifetime ratio between theory and experiment and penetration probability as a function of the radius parameter $r_{c}$ for the $\alpha$ decay of ${ }^{212}$ Po. Note that the latter quantity has been scaled up by a factor of $10^{10}$. The dotted line (unity) is given as a reference.

$$
V_{\alpha-\mathrm{Pb}}(r)=\frac{186}{1+\exp [(r-7.36) / 0.80]},
$$

where $V$ and $r$ are expressed in $\mathrm{MeV}$ and fm, respectively. The shape of this potential is quite different from the one of McFadden and Satchler [13], in spite of the close saturation value. A fair knowledge of the effective interaction (3) is a necessary ingredient to estimate the relative motion of the alpha particle prior to its tunneling decay from ${ }^{212}$ Po. Specifically, it either determines the wave function of the alpha particle near the nuclear surface in the $R$-matrix theory of Teichman and Wigner [14] (which we will apply here; cf. also Ref. [15]) or both bulk velocity and barrier profile in Gamow's. For clarity of presentation, we briefly review below the $R$-matrix approach to decay processes.

A dominant feature of reaction processes in which two nuclei collide at low energies to form a compound nucleus that subsequently disintegrates is the presence of resonances. In a time-independent framework, and assuming that the resonances do not overlap and are not close to thresholds, the $S$ matrix as a function of energy can be parametrized with a many-level Breit-Wigner formula

$$
S_{c c^{\prime}}(\mathcal{E})=e^{i\left(\delta_{c}+\delta_{c}^{\prime}\right)}\left[\delta_{c c^{\prime}}-i \sum_{n} \frac{\Gamma_{n, c^{1 / 2}}^{1 / 2}{ }_{n, c^{\prime}}}{\mathcal{E}-\omega_{n}}\right],
$$

where $\delta_{c}$ is the phase shift in channel $c$, the partial decay width $\Gamma_{n, c}$ is proportional to the probability that the resonance labeled by $n$ decays into the channel $c$, and $E_{n}=\operatorname{Re} \omega_{n}$ and $\Gamma_{n}=-2 \operatorname{Im} \omega_{n}$ are the positions and widths of the resonances. For isolated resonances only one term dominates Eq. (4), and the unitarity of the $S$ matrix requires that its residues $\Gamma_{n, c}^{1 / 2} \Gamma_{n, c^{\prime}}^{1 / 2}$ be real. In that case each $\Gamma_{n, c}$ can also be taken to be real, and $\Sigma_{c} \Gamma_{n, c}=\Gamma_{n}$. This situation does not occur frequently (e.g., among neutron-unstable states), and the physical meaning of the partial width can become questionable because they are truly complex quantities. However, for the $\alpha$ decay of low-lying states of heavy nuclei, this problem does not arise because the values of $\Gamma_{n} / E_{n}$ range between $10^{-10}$ and $10^{-30}$.

In the $R$-matrix theory one divides the configuration space of the $\alpha+$ daughter-nucleus system into two regions: an "internal region" $\mathcal{V}$ to which the compound state is restricted and an "external region" that encompasses the rest of the space. The boundary between the regions should be chosen such that all nuclear interactions and effects induced by the Pauli principle are only non-negligible in the internal region. In the external region the motion of the fragments is governed by the Coulomb interaction and the daughter nucleus and the $\alpha$ particle behave like a binary system moving outwards with asymptotic energy $E_{n}$. The solution inside the volume $\mathcal{V}$ satisfies a self-adjoint boundary condition on the surface $\mathcal{S}$ of $\mathcal{V}$. In the decay of spherical nuclei the surface $\mathcal{S}$ can be chosen to be a sphere of radius $r_{c}$ centered around the residual nucleus. The residues of the $S$ matrix can then be computed in terms of quantities evaluated on the surface $\mathcal{S}$, and one finds [15] that the lifetime for the decay is given by

$$
\tau\left(r_{c}\right)=\frac{\hbar}{2 P\left(r_{c}\right) \gamma^{2}\left(r_{c}\right)},
$$

where $P$ is the penetrability through the barrier and $\gamma$ is the reduced width amplitude.

We conclude with a concrete calculation of the lifetime for $\alpha$ decay of ${ }^{212}$ Po that exploits the procedures advocated here (this quantity has also been recently studied in detail using a different approach to the strong interaction in Ref. [16]). For our present purpose we have used the computer code GAMOW [17] to solve the Schrödinger equation for the $\alpha$ - $\mathrm{Pb}$ binding potential (3), imposing outgoing boundary conditions for the regular solutions. The eigenvalue corresponding to ten radial nodes turns out to be $8.96 \mathrm{MeV}$ with negligible imaginary part, a value remarkably close to the kinetic energy of the emitted alpha particle. We note that one could also in principle estimate the lifetime using that value of the imaginary part of the complex energy. However, as pointed out above, the ratio between the imaginary and real parts of the energy is so small that it cannot be calculated numerically with sufficient accuracy.

With the potential (3) and for $r \geqslant 9$ fm, only the tunneling through the Coulomb field is relevant. Since the resulting function $P\left(r_{c}\right)$ is strongly dependent on $r_{c}$, one may think that it is possible to obtain practically any value for the lifetime by adjusting the distance at which the penetration of the alpha cluster occurs. Actually, a consistency check on the theory is that $\tau\left(r_{c}\right)$ should be rather stationary for distances around the nuclear surface. The reduced width amplitude can be written as

$$
\gamma\left(r_{c}\right)=\sqrt{\frac{\hbar^{2} r_{c}}{2 \mu}} \Psi\left(r_{c}\right),
$$


where $\Psi$ is the wave function of the $\alpha$ particle. The expression for the lifetime quoted above does not take into account that only a fraction of the mother nucleus wave function corresponds to the motion of an $\alpha$ cluster around the daughter nucleus. This factor must be included to compare with experimentally measured lifetimes. A recent detailed microscopic calculation [2] shows that the $\alpha$ cluster spectroscopic factor is 0.025 . We adopt this value, which is also in good agreement [18] with the $\alpha$-formation amplitude extracted from a comparison between the Thomas theory [15] and experiment. We note that an older, large-scale shell model calculation [19] produced a value of $\sim 10^{-3}$ for that quantity.

In Fig. 2 we present the ratio between the experimental and calculated lifetimes as a function of the parameter $r_{c}$. We also show in the figure the penetrability $P$ as a function of distance. One can see that the theoretical calculation agrees extremely well with the experiment in a region close to the nuclear surface (the quality of this prediction may be best appreciated by pointing out that discrepancies between theory and experiment regarding the absolute values of $\alpha$-decay widths can easily span orders of magnitude). Notice that the lifetime results are indeed practically independent of $r_{c}$ over a large interval. While the penetration changes by nine orders of magnitude in the depicted range, the calculated ratio remains unity within, at worst, a factor of 2 .

The success of this simple calculation reveals the potential of the ideas introduced in this contribution. It should be noted that the prescription applies equally well to the investigation of more exotic emission processes that have also been contemplated (cf., e.g., Ref. [20] and references quoted therein). These involve the decay of heavier nucleon clusters such as carbon, oxygen, etc., which, at the microscopic dynamical level, would be even harder to approach. The information presently available for the elastic scattering of some of these systems may not be sufficient to extract a reliable energy dependence of the corresponding optical potentials. With the use of modern detection equipment, however, these measurements are not time consuming, as a recent determination of diagonal and off-diagonal matrix elements of the effective interactions has shown [21].

Discussions with T. Vertse are gratefully acknowledged. This work was supported in part by Grant No. DFG Bo 1109, EU Human Capital and Mobility program Grant No. ERBCHRX-CT92-0075, and Spanish CICYT Project No. PB92-0663.
[1] G. Gamow, Z. Phys. 51, 204 (1928).

[2] K. Varga, R. G. Lovas, and R. J. Liotta, Nucl. Phys. A550, 421 (1992).

[3] J. S. Bell and E. J. Squires, Phys. Rev. Lett. 3, 96 (1959).

[4] M. Namiki, Prog. Theor. Phys. (Kyoto) 23, 629 (1960).

[5] F. Michel, J. Albinski, P. Belery, Th. Delbar, Ch. Gregoire, B. Tasiaux, and G. Reidermester, Phys. Rev. C 28, 1904 (1983).

[6] Th. Delbar, Ch. Gregoire, G. Paic, R. Ceuleneer, F. Michel, R. Vanderpoorten, A. Budzanowski, H. Dabrowski, L. Freindl, K. Grotowski, S. Micek, R. Planeta, A. Strizalkowski, and K. A. Eberhard, Phys. Rev. C 18, 1237 (1978).

[7] B. Buck, C. B. Dover, and J. P. Vary, Phys. Rev. C 11, 1803 (1975).

[8] O. Akyüz and A. Winther, in Nuclear Structure and Heavy Ion Reactions, Proceedings of the International School of Physics “Enrico Fermi,', Course LXVII, Varenna, 1979, edited by R. A. Broglia, C. H. Dasso, and R. A. Ricci (North-Holland, Amsterdam, 1981).

[9] C. Mahaux, P. Bortignon, R. Broglia, and C. H. Dasso, Phys. Rep. C 120, 1 (1985).

[10] M. A. Nagarajan, C. Mahaux, and G. R. Satchler, Phys. Rev. Lett. 54, 1136 (1985).

[11] M. Nolte, H. Machner, and J. Bojowal, Phys. Rev. C 36, 1312 (1987).

[12] R. Broglia and A. Winther, in Heavy Ion Reactions (Addison Wesley, City, 1991).

[13] L. McFadden and G. R. Satchler, Nucl. Phys. 84, 177 (1966).
[14] T. Teichman and E. P. Wigner, Phys. Rev. 87, 123 (1952).

[15] R. G. Thomas, Prog. Theor. Phys. 12, 253 (1954).

[16] B. Buck, A. C. Merchant, and S. M. Perez, Phys. Rev. C 45, 2247 (1992).

[17] T. Vertse, K. F. Pal, and Z. Balogh, Comput. Phys. Commun. 27, 309 (1982).

[18] M. Iriondo, D. Jerrestam, and R. J. Liotta, Nucl. Phys. A454, 252 (1986)

[19] I. Tonozuka and A. Arima, Nucl. Phys. A323, 45 (1979).

[20] B. Buck and A. C. Merchant, Phys. Rev. C 39, 2097 (1989).

[21] J. de Boer, J. Fernández Niello, E. Hauber, E. Vogt, C. H. Dasso, M. Lozano, and G. Pollarolo, Nucl. Phys. 528, 242 (1991).

[22] A. M. Baxter, S. Hinds, R. H. Spear, T. H. Zabel, and R. Smith, Nucl. Phys. A369, 25 (1981).

[23] G. M. Hudson and R. H. Davis, Phys. Rev. C 9, 1521 (1974).

[24] A. R. Barnett and J. S. Lilley, Phys. Rev. C 9, 2010 (1974).

[25] J. S. Lilley, M. A. Franey, and D. H. Feng, Nucl. Phys. A342, 165 (1980).

[26] S. Shlomo, Y.-W. Lui, D. H. Youngblood, T. Udagawa, and T. Tamura, Phys. Rev. C 36, 1317 (1987).

[27] C. M. Perey and F. G. Perey, At. Nucl. Data Tables 17, 80 (1976).

[28] B. Bonin, N. Alamanos, B. Berthier, G. Bruge, H. Faraggi, D. Legrand, J. C. Lugol, W. Mittig, L. Papineau, A. I. Yavin, D. K. Scott, M. Levine, J. Arvieux, L. Farvacque, and M. Buenerd, Nucl. Phys. A430, 349 (1984). 